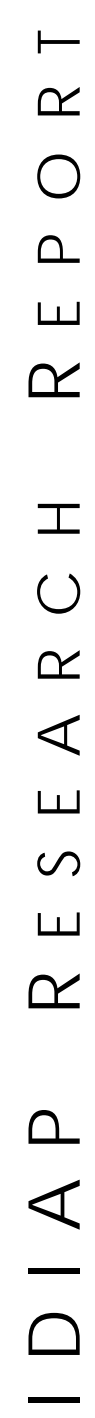

Martigny - Vala is - Suisse

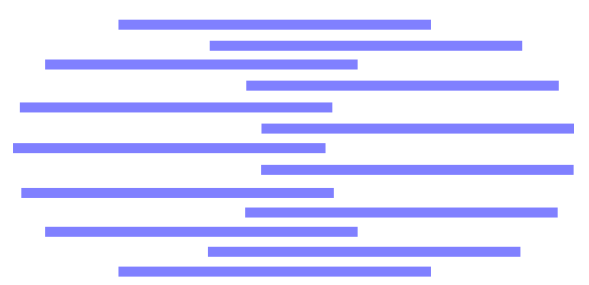

\title{
HANDWRITTEN Digit RECOGNITION WITH BINARY Optical PERCEPTRON
}

\author{
I. Saxena $^{\text {a }} \quad$ P. Moerland ${ }^{b} \quad$ E. Fiesler ${ }^{a}$ \\ A. Pourzand ${ }^{c}$ \\ IDIAP-RR 97-15
}

MAY 97

PUBLISHED IN

Proceedings of the International Conference on Artificial Neural Networks (ICANN'97), Lausanne, Switzerland, October 1997, 1253-1258

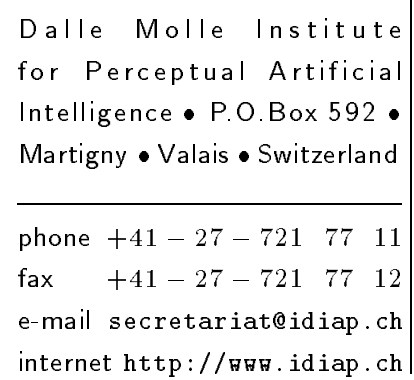

a Physical Optics Corporation, Gramercy Place, Torrance, CA 90501, U.S.A

b IDIAP, CP 592, CH-1920 Martigny, Switzerland. E-mail: Perry.Moerland@idiap.ch

c IMT, University of Neuchâtel, CH-2000 Neuchâtel, Switzerland 
IDIAP Research Report 97-15

\title{
Handwritten Digit Recognition with Binary Optical Perceptron
}

\author{
I. Saxena \\ P. Moerland \\ E. Fiesler \\ A. Pourzand
}

MAY 97

PUBLISHED IN

Proceedings of the International Conference on Artificial Neural Networks (ICANN'97), Lausanne, Switzerland, October 1997, 1253-1258

\begin{abstract}
Binary weights are favored in electronic and optical hardware implementations of neural networks as they lead to improved system speeds. Optical neural networks based on fast ferroelectric liquid crystal binary level devices can benefit from the many orders of magnitudes improved liquid crystal response times. An optimized learning algorithm for all-positive perceptrons is simulated on a limited data set of hand-written digits and the resultant network implemented optically. First, gray-scale and then binary inputs and weights are used in recall mode. On comparing the results for the example data set, the binarized inputs and weights network shows almost no loss in performance.
\end{abstract}




\section{Introduction}

In hardware implementations of neural networks, it is attractive to use binary weights and inputs. In electronic implementations, reduction of chip area, reduced computation and improved system speed drive the motivation to enable the use of binary or a minimum number of discrete weights [1]. Therefore, training algorithms are sought that enable to successfully implement binary level devices in neural networks.

Optical neural network implementations can benefit from the faster speed inherent in the free space propagation of information as light. The limitations that exist in 2-D electronic implementations are further overcome in 3-dimensional free space optical interconnections, allowing much higher interconnectivity. In optical implementations, where inputs and weights are implemented by liquid crystal television (LCTV) screens, and non-linear thresholding by LCLVs (for example, [9]), the processing speed is limited by the liquid crystal response time. Typically nematic liquid crystal based devices are used which have frame rates of $50 \mathrm{~Hz}$ and non-linear responses which allow several gray level weights and/or inputs to be implemented. On the other hand, higher frame rates several orders of magnitude faster, up to a few $100 \mathrm{kHz}$ [2] are offered by emerging ferro-electric liquid-crystal devices which have a limited number of (binary) weighting levels. From a device point of view, ferro-electric liquid crystal (FE-LC) devices merge well with VLSI techniques and can be integrated in a hybrid of passive and active devices to provide compact, efficient device geometries.

We present results of our work in the course of developing useful adaptive optical multilayer perceptrons (MLPs). The success of this approach rests on the development of practical devices and techniques which reliably implement a large number of dense free-space optical interconnects and training rules optimized to benefit from practical device characteristics. These two areas affecting implementation and whether they can be consolidated in a useful neural network application is explored here from the perspective of binary level devices. This is illustrated by some experimental results of recall on a optical perceptron, based on an optical system described earlier [9]. Recall is done for a small subset of handwritten digits and illustrates that binarized inputs and weights can in principle be sufficient for successful performance.

\section{Description of Optical Multilayer Perceptron and Training Algorithm}

The optical multilayer perceptron is based mostly on available hardware with the input layer and programmable weights for the interconnects between layers being implemented by liquid crystal television (LCTV) screens and the hidden layer and the output layer by liquid crystal light valves (LCLVs), as described in [9]. A computer is used for the computationally intensive parts of the learning algorithm.

\subsection{Optical System}

The first matrix-vector multiplier or MVM takes as input a $16^{*} 16$ array of 4-bit precision numbers, generates $16^{*} 16$ copies of the input array, and multiplies each copy by a $16^{*} 16$ array of 4 -bit weight values on a point-by-point basis employing one pixel of the weight screen, the LCTV2, per interconnect channel [3]. The chosen LCTV screens used for these results have a complexity of $480^{*} 440$ pixels (Seiko Epson video projector, model \#VPJ-2000), which are, sufficient for the full (16*16) complexity multiplier except for the problem of exact pixel address. An alternative LCTV screen can now provide exact pixel address for future planned experiments. The hidden layer module takes a $16^{*} 16$ array of data blocks as input and outputs a $16^{*} 16$ array of spots each of which represents the integrated intensity in a data block transformed according to the nonlinear transfer characteristic of LCLV1. The integration will be performed by a lens-let array. The lens-lets in the array have a repeated spacing equal to the spacing of the sub-arrays on LCTV2 and a focal length of several mm. Results on the fan-in shall be available shortly. The result of the integration will be a $16^{*} 16$ array of spots 


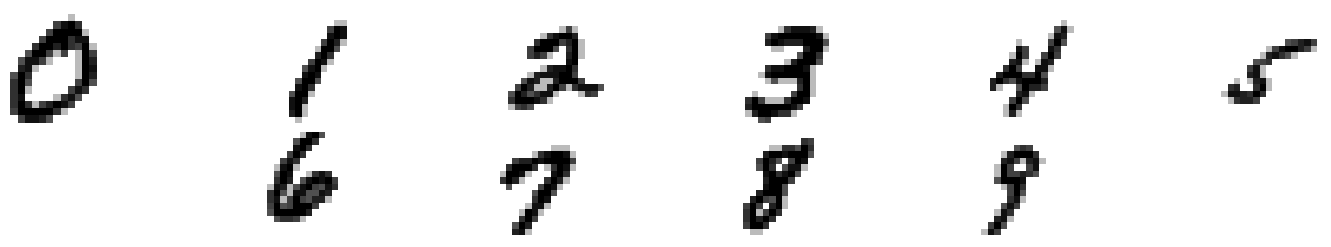

Figure 1: Gray-scale Test Inputs: A set of hand-written digits (16 gray levels).

on the photo-conductor of LCLV1. These spots activate the liquid crystal (LC) layer, changing its transmission nonlinearly as a function of incident light intensity. The activation of the LC layer will be read out by a $16^{*} 16$ array of spots from a helium neon laser with a wavelength at which the photoconductor is transparent. Using a focussed array of readout beams to optimize the light budget is prevented here as it results in a reduction of the contrast ratio as compared to that with a collimated read beam.

\subsection{Training Algorithm Optimization}

There are three important aspects of algorithm development that we have addressed which now allow optical multilayer neural network implementations [7]. These concern the questions on the usefulness of the non-linearities offered by LCLVs, of implementing the well-known only-positive-weights limitation of incoherent optical processing systems, and determining the (minimum) number of possible weight discretization levels for a particular optical neural network to overcome analog hardware non-idealities as implemented by LCTVs [8]. Empirical studies on all-positive neural networks without the possibility of subtraction (of bias thresholds, for instance) strongly indicate that such networks are not useful (due to monotonicity). The possibility of subtraction has therefore been included in our training algorithm whereby all positive weights are implemented by including a subtractive-feedback to enable useful optical neural networks [7]. The training of the optical neural network is performed by the weight discretization and update algorithm described in [4].

\section{Experiment and Results}

\begin{tabular}{|c|r|r|r|r|r|r|r|r|r|r|}
\hline $\begin{array}{c}\text { Input digit } \\
\text { Pattern }\end{array}$ & 0 & 1 & 2 & 3 & 4 & 5 & 6 & 7 & 8 & 9 \\
\hline Computed & 1.0 & 0.65 & 0.79 & 0.89 & 0.72 & 0.53 & 0.82 & 0.77 & 0.75 & 0.56 \\
Optical: Gray-scale & 1.0 & 0.83 & 0.94 & 0.86 & 0.81 & 0.56 & 0.74 & 0.72 & 0.72 & 0.48 \\
Optical: Binary & 1.0 & 0.91 & 0.32 & 0.55 & 0.76 & 0.64 & 0.79 & 0.82 & 0.83 & 0.45 \\
\hline
\end{tabular}

Table 1: ONN Recall performance: Comparison of simulated and optical results prior to thresholding, showing the normalized outputs at the winner neurons.

Experiments have been performed with the set-up described in the previous section on a twolayer ONN or optical perceptron. Training of the perceptron using the training algorithm (including weight discretization, compensation for the LCLV non-linearities, and subtractive feedback for allpositive weights) described in section 2.2 was simulated on a computer. A subset of 50 digits from the handwritten digit data set of the NIST database [5] was used to train the perceptron and the resultant weights were implemented on the LCTV of the optical perceptron for testing recall with only 10 of the 50 digits. A first experiment used weights with 8 discrete levels and 16 (equidistant) gray levels for the inputs (see figure 1 for the images of the ten digits used for recall). The first two rows of 

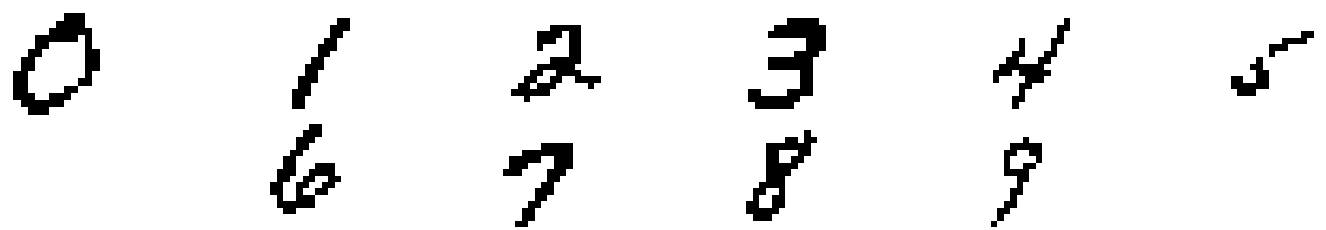

Figure 2: Binary Test Inputs: A binarized set of hand-written digits.

Table 1 show the recall results of the computer simulated values as compared to those obtained with the optical system using the several gray level capability of the LCTVs to represent the weights. The experimental results on the optical perceptron show quite good agreement with the computed values (within $20 \%$ ). Next, the training on computer was repeated with binarized inputs (see figure 2 for the images of the ten digits used for recall) and binary weight values. The third row of Table 1 shows the corresponding recall results for binarized (with a hard threshold) inputs and binary weights to simulate the effect of FE-LCTV binary implementations. Successful discrimination by the binarized (input and weight) neural network was obtained as can be seen in Table 2, where unique outputs with high signal-to-noise ratio are obtained with the binarized data.

\begin{tabular}{|c|r|r|r|r|r|r|r|r|r|r|}
\hline Input digit & 0 & 1 & 2 & 3 & 4 & 5 & 6 & 7 & 8 & 9 \\
\hline 0 & 1.0 & 0.00 & 0.00 & 0.00 & 0.00 & 0.00 & 0.00 & 0.00 & 0.00 & 0.00 \\
1 & 0.00 & 0.91 & 0.00 & 0.00 & 0.00 & 0.00 & 0.00 & 0.00 & 0.00 & 0.02 \\
2 & 0.00 & 0.00 & 0.32 & 0.00 & 0.00 & 0.00 & 0.00 & 0.00 & 0.00 & 0.00 \\
3 & 0.00 & 0.00 & 0.00 & 0.55 & 0.00 & 0.00 & 0.00 & 0.00 & 0.00 & 0.00 \\
4 & 0.00 & 0.00 & 0.00 & 0.00 & 0.76 & 0.00 & 0.00 & 0.00 & 0.00 & 0.00 \\
5 & 0.00 & 0.00 & 0.00 & 0.00 & 0.00 & 0.64 & 0.00 & 0.00 & 0.00 & 0.00 \\
6 & 0.00 & 0.00 & 0.00 & 0.00 & 0.00 & 0.00 & 0.79 & 0.00 & 0.00 & 0.00 \\
7 & 0.00 & 0.00 & 0.00 & 0.00 & 0.00 & 0.00 & 0.00 & 0.82 & 0.00 & 0.00 \\
8 & 0.00 & 0.00 & 0.00 & 0.00 & 0.00 & 0.00 & 0.00 & 0.00 & 0.83 & 0.00 \\
9 & 0.00 & 0.00 & 0.00 & 0.00 & 0.00 & 0.00 & 0.00 & 0.00 & 0.00 & 0.45 \\
\hline
\end{tabular}

Table 2: The full output layer for recall with a binary optical perceptron.

\section{Summary}

Binarized inputs and weights are implemented in LCTV screens having gray-level capability, to predict the behaviour of perceptron algorithms when implemented with emergent faster FE-LCTV modulators. Satisfactory agreement (however with a limited data set training) indicates a possible capability of incorporating faster binary level devices in optical neural networks. This is to be verified with larger data set experiments.

Investigations are also required to examine the applicability of the LCLV compensation theorem [6][10] to FE-LC devices as non-linear optical thresholding elements in optical neural networks, to evaluate whether one can take advantage of the faster response times of FE-LC devices. This theorem provides a clear relationship between training parameters that enables compensating for non-standard gains of the non-linear thresholding functions, and could therefore compensate for the sharp response of FE-LCs. ${ }^{1}$

\footnotetext{
${ }^{1}$ An increase of the gain with a factor $\beta$ can therefore be compensated for by dividing the initial weights by $\beta$ and the learning rate by $\beta^{2}$.
} 


\section{Acknowledgments}

This work was partially supported by the Swiss National Science Foundation (FN: 43220.95).

\section{References}

[1] S. Abramson, D. Saad, and E. Marom, "Training a Neural Network with Ternary Weights Using the CHIR Algorithm," IEEE Transactions on Neural Networks, 4(6), 997-1000, (1993).

[2] S. E. Broomfield, M. A. A. Neil, and E. G. S. Paige, "Programmable multiple-level phase modulation that uses ferroelectric liquid crystal spatial light modulation," Applied Optics, 34(29), $6652-6665$ (1995).

[3] N. Collings, A. R. Pourzand, and R. Volkel, "Construction of a programmable multilayer analogue neural network using space invariant interconnects," in SPIE Vol. 2565, pp. 40-47 (1995).

[4] E. Fiesler, A. Choudry, and H. J. Caulfield, "A Weight Discretization Paradigm for Optical Neural Networks," in Proceedings of the International Congress on Optical Science and Engineering, vol. SPIE 1281, pp. 164-173, SPIE, Bellingham, Washington (1990).

[5] M. D. Garris and R. A. Wilkinson, data from NIST Special Database 3, National Institute of Standards and Technology (1992).

[6] P. Moerland, E. Fiesler, and I. Saxena, "Incorporation of Liquid-Crystal Light Valve Non-Linearities in Optical Multilayer Neural Networks," Applied Optics, 35(26), 5301-5307 (1996). P. Moerland, E. Fiesler, and I. Saxena.

[7] P. Moerland, E. Fiesler, and I. Saxena, "Discrete All-Positive Multilayer Perceptrons for Optical Implementations," IDIAP-RR 97-02, IDIAP, Martigny, Switzerland, February 1997 (accepted for publication in Optical Engineering).

[8] M. G. Robinson and K. M. Johnson, "Noise Analysis of Polarization-Based Optoelectronic Connectionist Machines," Applied Optics, 31(2), 263-272 (1992).

[9] I. Saxena, P. Moerland, E. Fiesler, A. R. Pourzand, N. Collings, "An Optical Thresholding Perceptron," in Proceedings of the Workshop on Optics and Computer Science, Geneva, Switzerland (1997).

[10] G. Thimm, P. Moerland, and E. Fiesler, "The Interchangeability of Learning Rate and Gain in Backpropagation Neural Networks," Neural Computation, 8(2), 451-460 (1996). 